\title{
Prevalênciade tuberculose pulmonar no Brasil: uma revisão bibliográfica
}

\author{
Prevalence of pulmonary tuberculosis in Brazil: \\ a bibliographic review
}

\author{
Laís da Silva Pacheco ${ }^{1}$ \\ Laura Vicedo Jacociunas ${ }^{2}$
}

\section{RESUMO}

A tuberculose é uma doença infectocontagiosa que afeta frequentemente os pulmões, cujo agente causador é o Mycobacterium tuberculosis. É transmitida pela fala, tosse ou espirro de um portador bacilífero. Possui bom prognóstico e cura quando diagnosticada e tratada precocemente. Segundo a Organização Mundial da Saúde, cerca de um quarto da população mundial possui infecção latente por este microrganismo. Está entre as dez principais causas de morte no mundo e a principal causa por um único agente transmissor. 0 estudo teve como objetivo realizar uma revisão de literatura sobre a prevalência de tuberculose no Brasil. Adotou como metodologia e ferramenta embasadora, a utilização de materiais já publicados, livros, artigos científicos, publicações periódicas e documentos oficiais. Trabalhos que constavam na lista de referências dos artigos encontrados foram utilizados como leitura suplementar. Os termos utilizados para busca foram: bacilo de Koch, cultura, diagnóstico, prevalência, micobactérias, Mycobacterium tuberculosis, radiografia de tórax, teste rápido molecular,tuberculose pulmonar, tratamento, bem como a combinação entre eles nos idiomas: português, inglês e espanhol. Não houve limite de ano para busca de publicação. Os artigos foram selecionados pelo título e resumos. Apresenta como resultadosaumento na média de prevalência nacional em 2019, seguido por queda acentuada em 2020. Há um predomínio de acometimento pela doença no sexo masculino, além disso, ela se apresenta mais prevalente nas regiões Norte e Sudeste. Conclui-se que o Brasil apresenta tendência de queda nos índices de tuberculose, mas ainda possui alta prevalência, dentre outros índices relevantes.

\section{PALAVRAS-CHAVE}

Tuberculose;Tuberculose pulmonar;Mycobacterium tuberculosis; Prevalência; Epidemiologia.

\footnotetext{
${ }^{1}$ Acadêmica do Curso de Biomedicina do Centro Universitário Metodista - IPA.

${ }^{2}$ Biomédica, Especialista em Toxicologia Aplicada, mestre e Doutora em Genética e Toxicologia Aplicada. Coordenadora do Curso de Biomedicina do Centro Universitários Metodista IPA.
} 


\begin{abstract}
Tuberculosis is an infectious contagious disease that frequently affects the lungs, whose causative agent is $M y c o-$ bacterium tuberculosis. It is transmitted by the speech, coughing or sneezing of a bacillus carrier. It has a good prognosis and cure when diagnosed and treated early. According to the World Health Organization, about a quarter of the world population has latent infection by this microorganism. It is among the ten leading causes of death in the world and the leading cause of a single causative agente. It aims to conduct a literature review on the prevalence of the pulmonary tuberculosis in Brazil. It adopts as a methodology the use as a supporting tool of already published materials, books, scientific articles, and periodical publications and official documents. Works that were in the reference list of the articles found were used as supplementary reading. The terms used for the search were: Koch's bacillus, culture, diagnosis, prevalence, mycobacteria, Mycobacterium tuberculosis, chest X-ray, rapid molecular test, pulmonary tuberculosis, treatment, as well as their combination in the languages: Portuguese, English and Spanish. There was no year limit for search for publication. Articles were selected by title and abstracts. Results show an increase in the nacional average incidence in 2019, followed by a sharp drop in 2020. There is a predominance of involvement by the disease in males, in addition, it has a higher prevalence in the North and Southeast regions. It is concluded that Brazil has a downward trend in tuberculosis rates, but still has a high prevalence, among other relevant rates.
\end{abstract}

\title{
KEYWORDS
}

Tuberculosis; Pulmonary tuberculosis; Mycobacterium tuberculosis; Prevalence; Epidemiology. 


\section{INTRODUÇÃo}

A tuberculose (TB) é uma doença infectocontagiosa causada principalmente pelo Mycobacterium tuberculosis (MTB)ou Bacilo de Koch (BK). Ela afeta frequentemente os pulmões, mas pode acometer outros órgãos quando em forma extrapulmonar, que ocorre principalmente em casos de imunocomprometimento grave de pacientes portadores de Síndrome da Imunodeficiência adquirida (SIDA; em inglês: acquired immunodeficiency syndrome, AIDS). (WHO, 2020a)

A TB pode ser causada por qualquer uma das espécies que integram o complexo Mycobacterium tuberculosis: M. tuberculosis, M. bovis, M. africanum, M. canetti, $M$. microti, M. pinnipedii e M. caprae. Entretanto, o principal agente etiológico da doença é o MTB, um microrganismo aeróbio, delgado, ligeiramente curvo, medindo de 0,5 a $3 \mu \mathrm{m}$. É considerado um bacilo álcool-ácido resistente (BAAR), com parede celular rica em lipídios (ácidos micólicos e arabinogalactano), que lhe conferem baixa permeabilidade, reduzindo a efetividade da maior parte dos antibióticos, facilitando sua sobrevida nos macrófagos. (BRASIL, 2019a; ROSSMAN; MACGREGOR, 1995)

Segundo evidências arqueológicas e históricas, os primeiros casos de acometimento por tuberculose ocorreram há mais de 5000 anos a.C. em múmias egípcias. Estas apresentavam tecido contendo o DNA (Ácido Desoxirribonucleico)do Mycobacterium tuberculosis, além de um comprometimento vertebral (o Mal de Pott) tipicamente causado pelo microrganismo. (DANIEL, 2000).

Mesmo que comprovada na América pré-colombiana, relatos históricos da época indicam que não havia TB entre os nativos brasileiros antes da chegada dos portugueses. Desta forma, a doença foi introduzida pelos colonizadores europeus, principalmente padres jesuítas, que mantinham contato frequente com os nativos, facilitando a disseminação da doença. As condições a que eram expostos os africanos após o tráfico negreiro também propiciou a expansão da TB, assim em 1855 no Rio de Janeiro, um quinto dos doentes internados nos hospitais sofria pela doença. (VERONESI, FOCACCIA, 2015)

A transmissão ocorre por via aérea através da fala, tosse ou espirro de portadores bacilíferos da doença pulmonar ou laríngea. 0 termo bacilífero se refere aos pacientes que apresentam baciloscopia de escarro positiva. Estes são a principal fonte de disseminação da doença, e assim a busca ativa por sintomáticos respiratórios (SR) torna-se uma medida importante para interromper a cadeia de transmissão. (BRASIL, 2019a)

Os portadores de tuberculose ativa produzem as gotículas de Flügge, que ao serem exaladas tornam-se secas e se transformam em partículas menores (núcleos de Wells de $<5-10 \mu \mathrm{m})$. Estes por sua vez, contém de um a dois bacilos e podem ficar suspensos no ar por horas, sendo capazes de alcançar os alvéolos podendo se multiplicar e provocar a primo-infecção. (CARDONA, 2018; CDC, 2012)

Com o diagnóstico e o tratamento corretos, a maior parte das pessoas que desenvolvem tuberculose podem ser curadas. A tosse é o principal sintoma sugestivo de TB, principalmente quando apresentada por 3 semanas ou mais. Desta forma, a busca ativa por SR é considerada a principal forma de diagnóstico precoce da doença. (WHO, 2017; BRASIL, 2019a)

A baciloscopia de escarro é um importante método para diagnóstico e controle de tuberculose no Brasil. Ela consiste na identificação do MTB utilizando-se da metodologia de Ziehl-Neelsen, com o esfregaço do escarro sendo fixado e corado em lâmina para leitura posterior. 0 resultado é expresso em ?cruzes? conforme a quantidade de bacilos encontrados a partir da amostra. (BRASIL, 2019c). A cultura é o método padrão-ouro para diagnóstico de tuberculose, isto devido a sua alta sensibilidade e especificidade. Porém, o MTB tem como característica crescimento lento, necessitando de um período de 4 a 8 semanas para a confirmação do resultado, podendo retardar o início do tratamento da doença. (OLIVEIRA et al., 2018)

Outros métodos de diagnóstico também são aplicados, como a prova tuberculínica que auxilia no diagnóstico da TB em pessoas não vacinadas com BCG (Bacilo de Calmette e Guérin). (STATENS SERUM INSTITUT, 2020). A radiografia é um exame complementar utilizado no diagnóstico da tuberculose, pois a doença pode apresentar diversos padrões radiológicos. (SILVA et al., 2021b). 0 gene Xpert MTB/RIF é o único teste rápido molecular recomendado pela OMS para o diagnóstico de tuberculose. Sua metodologia se baseia na reação em cadeia da polimerase (Polymerase Chain Reaction- PCR) para detectar o DNA do Mycobacterium tuberculosis e simultaneamente a resistência à rifampicina. (WHO, 2018) 
0 tratamento da tuberculose tem como objetivo curar e reduzir rapidamente a transmissão da doença. Por isso é necessário utilizar fármacos com capacidade de reduzir rapidamente a população bacilar, prevenir a seleção de cepas resistentes e esterilizar a lesão afim de prevenir a recidiva da doença. (SOTGIU et al., 2016)

Segundo a Organização Mundial da Saúde (OMS), cerca de um quarto da população mundial está infectada pelo Mycobacterium tuberculosis e há um risco de 5\% a $10 \%$, aproximadamente 2 bilhões de pessoas, desenvolverem a doença ativa durante sua vida. Entretanto, pessoas que vivem com o vírus da imunodeficiência humana (VIH ou HIV, do inglês Human Immunodeficiency Virus) ou com fatores de risco como desnutrição, diabetes ou tabagismo possuem uma probabilidade ainda maior de desenvolver TB. (WHO, 2020b)

No ano de 2015 segundo a OMS (2016), mundialmente, cerca de 10,4 milhões de pessoas adoeceram por TB, e destas 1,8 milhão morreram. Neste mesmo anoa região das Américas representou 3\% da carga mundial da doença, sendo o Brasil o país a ter a maior porcentagem de novos casos, em torno de 33\%. Além disso, foi classificado como um dos 22 países com maior carga de TB no mundo. 0 percentual de detecção de TB no Brasil em 2015 foi de 87,0\%. (WHO, 2016; WHO, 2017)

Embora tenha-se notado queda entre os anos 2010 e 2016, houve aumento no coeficiente de TB no Brasil nos anos de 2017 e 2018. Em 2019 foram diagnosticados 73.864 novos casos da doença, correspondendo a um coeficiente de prevalência de 35,0 casos/100 mil habitantes. (BRASIL, 2020a)

Em dezembro de 2019 na cidade de Wuhan na China ocorreu o início da pandemia de Covid-19, doença causada pelo coronavírus SARS-CoV-2 que pode gerar pneumonia grave além de outras complicações. Esta enfermidade associada a TB apresenta grande potencial de morbidade e mortalidade, além da possibilidade de causar impactos referentes ao diagnóstico e tratamento da doença. (MACIEL, JÚNIOR, DALCOLMO, 2020; SILVA et al., 2021a)

O Brasil encontra-se distante dos objetivos preconizados pela OMS até 2035, na chamada Estratégia pelo fim da Tuberculose, que tem como principais metas reduzir o coeficiente de prevalência para menos de 10 casos por 100 mil habitantes, além de reduzir o número de óbitos pela doença. (MACIEL, JÚNIOR, DALCOLMO, 2020)
Diante dos desfechos citados anteriormente referente a tuberculose, e a fim de contribuir para futuras pesquisas e políticas públicas e auxiliar políticas de prevenção, esta revisão tem como objetivo destacar os principais índices sobre a prevalência de tuberculose no Brasil.

\section{METODOLOGIA}

O estudo foi realizado através de uma revisão da literatura, utilizando como ferramenta embasadora materiais já publicados sobre o tema, livros, artigos científicos, e publicações periódicas. Foram revisadas as bases de dados PubMed (National Library of Medicine), Scielo (Sientific Eletronic Library Online), Lilacs (Literatura Latino Americana e do Caribe em Ciências da Saúde), além de documentos oficiais como dados da Organização Mundial da Saúde, e Ministério da Saúde. Outros trabalhos que constavam na lista de referências dos artigos encontrados foram utilizados como leitura suplementar. Os termos utilizados para busca foram: bacilo de Koch, cultura, diagnóstico, incidência, micobactérias, Mycobacterium tuberculosis, radiografia de tórax, teste rápido molecular, tuberculose pulmonar, tratamento, bem como a combinação entre eles nos idiomas: português, inglês e espanhol. Não houve limite de ano para busca de publicação. Em um primeiro momento, os artigos foram selecionados pelo título e resumos.

\section{RESULTADOS E DISCUSSÃO}

A tuberculose continua sendo considerada mundialmente um importante problema de saúde pública. No ano de 2018 foi classificada como a principal causa de morte de um único agente infeccioso, além disso, cerca de 10 milhões de pessoas adoeceram e 1,5 milhão morreram por conta da doença. A ocorrência desta enfermidade aponta para fatores socioeconômicos, uma vez que afeta de maneira desproporcional pessoas do sexo masculino, adultos jovens e países de baixa renda. (WHO, 2019)

Segundo a OMS (2020b), no ano de 2019, em torno de 10 milhões de pessoas desenvolveram TB e 1,2 milhão morreram em decorrência da doença. 0 Brasil permanece entre os 30 países com alta carga de TB e de 
coinfecção TB-HIV, sendo assim considerado prioritário para o controle da enfermidade.

No Brasil, em 2019, houve aumento no coeficiente de prevalência (por 100 mil habitantes) em relação aos anos anteriores. Contudo, é possível perceber que em 2020, durante a pandemia de COVID-19, houve queda na prevalência de TB comparando-a com dados dos dois anos anteriores: 2018 e 2019. (BRASIL, 2021) (Figura 1).É possível que esta redução esteja relacionada ao distanciamento social e ao contínuo uso de máscaras pela população, medidas preventivas e estratégias de saúde pública que foram adotadas desde março de 2020 justamente devido a pandemia do Coronavírus, causador da COVID-19 (BRASIL, 2020c).

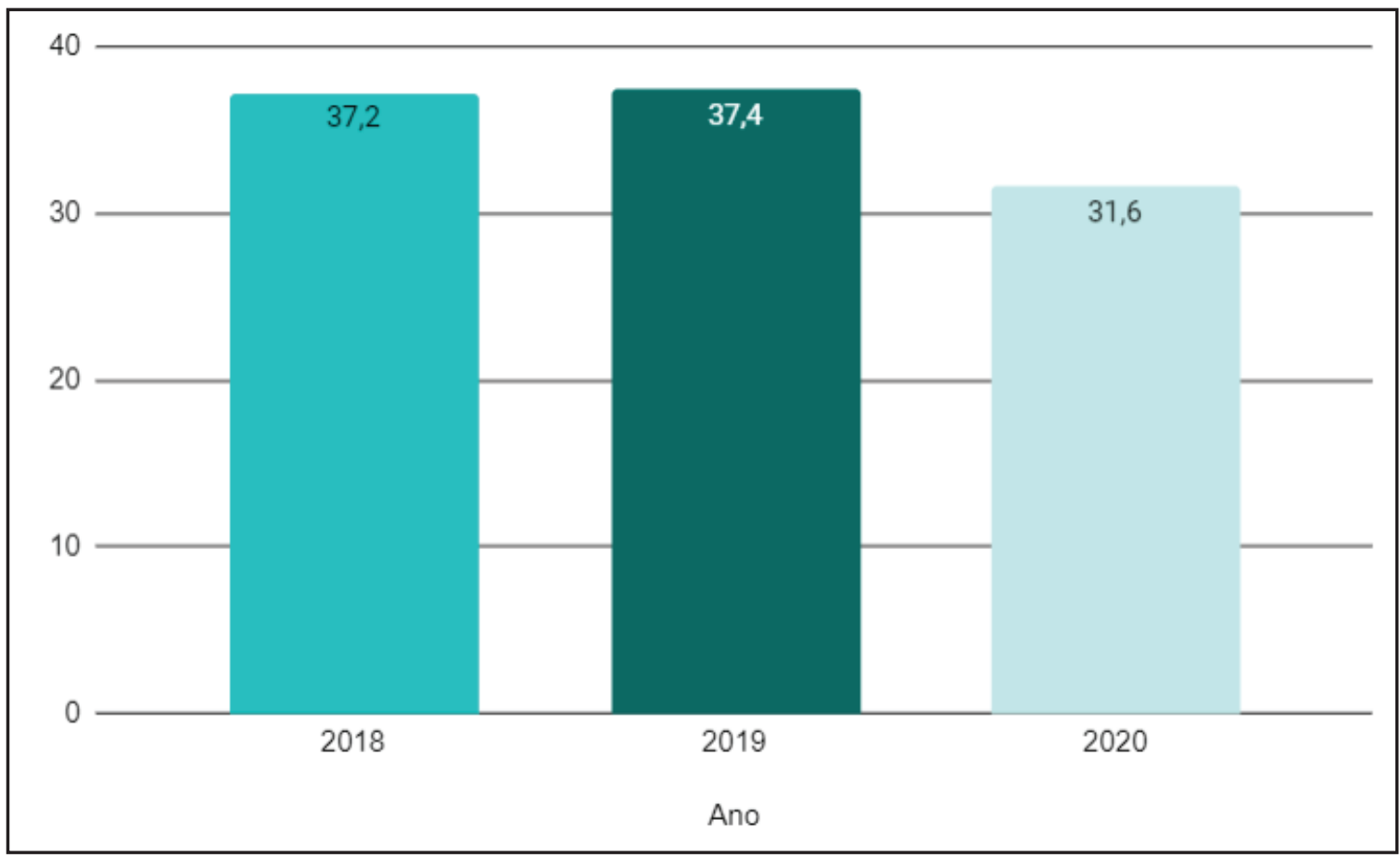

Figura 1 - Coeficiente de prevalência/100mil hab. de tuberculose.Brasil, 2018 a 2020.

Fonte: Sistema de Informação de Agravos de Notificação/Secretarias Estaduais de Saúde/Ministério da Saúde; Instituto Brasileiro de Geografia e Estatística. 2021.

Ainda em 2019, segundo a OMS (2020b), 56\% das pessoas acima de 15 anos que desenvolveram tuberculose eram do sexo masculino, 32\% do sexo feminino e 12\% eram crianças ( $<15$ anos). Dentre todos os afetados pela doença, 8,6\% eram pessoas vivendo com HIV.

De acordo com dados do boletim epidemiológico brasileiro de 2021, no período de 2011 a 2020, 69,0\% dos casos novos de tuberculose, em torno de 46.130, ocorreram em pessoas do sexo masculino. Na Figura 2 de 2020, sobre o coeficiente de casos novos de TB por região e sexo, é possível perceber que pessoas do sexo masculino são as mais afetadas por TB. Além disso, a raça/cor preta/parda representou a maior prevalência, variando de 60,2\% a 66,8\%. 


\section{Feminino Masculino}

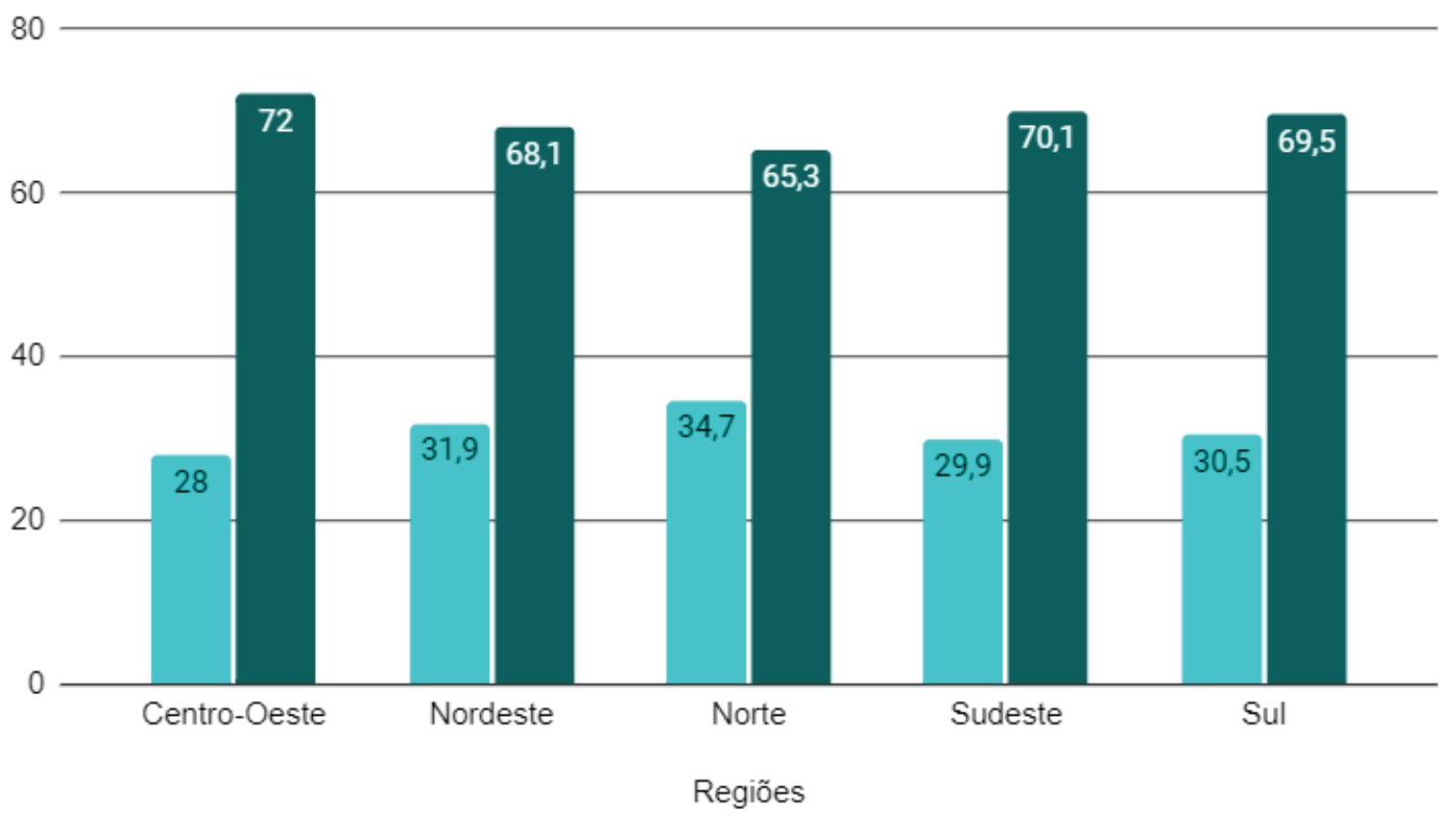

Figura 2 - Coeficiente de casos novos/100 mil hab. de tuberculose por regiões e sexo. Brasil, 2020.

Fonte: Sistema de Informação de Agravos de Notificação/Secretarias Estaduais de Saúde/Ministério da Saúde; Instituto Brasileiro de Geografia e Estatística. 2021.

Além do predomínio de acometimento por TB no sexo masculino, é possível observar uma menor adesão ao tratamento levando, consequentemente, ao abandono deste. Marques et al. (2018), realizou uma pesquisa no período compreendido entre os anos de 2001 a 2015 e observou maior prevalência de TB em pessoas do sexo masculino. Resultados semelhantes foram observados no estudo de Silva (2017), que relatou maior prevalência de TB em pessoas do sexo masculino, além disso, menor adesão ao tratamento, também levando ao abandono. Da mesma forma, pesquisa desenvolvida por Santos et al. (2021) verificou altas taxas de abandono no tratamento, corroborando com o presente estudo.

Na estratificação por regiões do Brasil é possível perceber no mapa (Figura 3) que a região brasileira com a maior prevalência de tuberculose é a região Norte com 43,0 casos por 100 mil hab. A região Sudeste apresenta-se em segundo lugar com 34,4 , seguida pela região Nordeste com 30,1. E as duas regiões com menor coeficiente de prevalência são região Sul e região Centro-Oeste, com 26,0 e 18,0, respectivamente. (BRASIL, 2021)
De acordo com os dados relatados na figura 3, pelo menos duas regiões brasileiras apresentaram no ano de 2020 um coeficiente de prevalência maior do que o coeficiente de prevalência de média nacional, demonstrado na figura 1. Estas regiões foram: norte e sudeste. Estes dados corroboram com as tendências epidemiológicas avaliadas nos últimos anos e apresentadas por Cortez et al. (2021) na qual os dados epidemiológicos analisados pelos autores no período de 2006 a 2015 estão em consonância com o presente estudo, demonstrando maior prevalência de TB nas regiões norte e sudeste. $\mathrm{Na}$ região Norte, as elevadas taxas de incidência podem estar ligadas à precariedade no sistema público de saúde que atrasam o diagnóstico mantendo assim a cadeia de transmissão. Já a região Sudeste é a mais populosa do Brasil e isso possui uma relação direta com a transmissibilidade da tuberculose, fato que influencia as taxas de incidência da doença. (Cortez et al.; 2021)

De forma global a ocorrência da TB aponta para fatores socioeconômicos, pois afeta de maneira desproporcional pessoas do sexo masculino, jovens e principalmente países de baixa renda. 


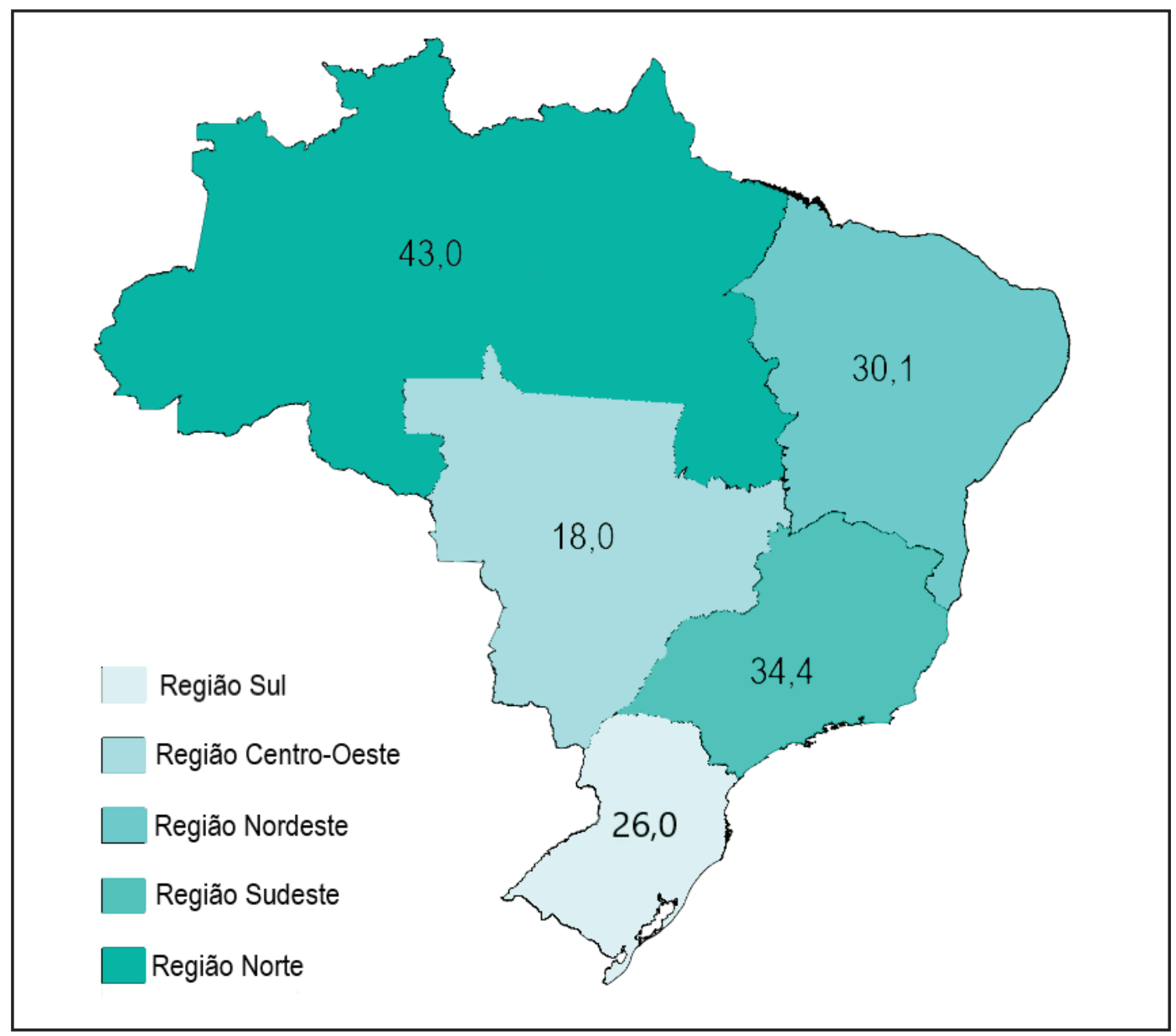

Figura 3 - Coeficiente de prevalência de casos/100 mil hab. de tuberculose por região. Brasil, 2020.

Fonte: Sistema de Informação de Agravos de Notificação/Secretarias Estaduais de Saúde/Ministério da Saúde; Instituto Brasileiro de Geografia e Estatística. 2021.

\section{CONCLUSÃO}

O desenvolvimento do presente estudo possibilitou a realização de uma revisão de literatura sobre a tuberculose e o seu coeficiente de prevalênciano Brasil. Além da comparação dos coeficientes gerais dos últimos 3 anos no país, foi apresentado um panorama dos índices globais da doença.

Apesar de ter apresentado uma queda acentuada no ano de 2020 no Brasil, em meio à pandemia de Covid-19, a tuberculose permanece sendo um problema de saúde pública bastante relevante, pois além do grande potencial de morbidade e mortalidade da doença quando associada à Covid-19, os índices de mortalidade e prevalência de TB ainda se mostram distantes do preconizado pela OMS. Além de aspectos sociais e econômicos, a assistência à tuberculose envolve iniciativas governamentais, diagnóstico precoce e tratamento adequado. 


\section{REFERÊNCIAS}

BERGEL, F.S.; GOUVEIA, N. Manual de recomendações para o controle da tuberculose no Brasil. Retornos frequentes como nova estratégia para adesão ao tratamento de tuberculose. Rev. Saúde Pública, v.31, n.9, 2013.

BERTOLOZZI, M. R., TAKAHASHI, R. F., HINO, P., LITVOC, M., \& FRANÇA, F. O. de S. (2014). 0 controle da tuberculose: um desafio para a saúde pública. Revista De Medicina, 93(2), 83-89.

BLOOM, B.R.; ATUN, R.; COHEN, T.; DYE, C.; FRASER, H.; GOMEZ, G. B.; KNIGHT, G.; MURRAY, M.; NARDELL, E.; RUBIN, E.; SALOMON, J.; VASSALL, A.; VOLCHENKOV, G.; WHITE, R.; WILSON, D.; YADAV, P. Em Major Infectious Diseases; HOLMES, K.K.; BERTOZZI, S.; BLOOM, B. R.; Jha, P., eds.; Washington (DC): The International Bank for Reconstruction and Development / The World Bank, 2017, cap. 11.

BRASIL. Ministério da Saúde. Secretaria de Atenção à Saúde. Departamento de Atenção Básica. Cadernos de Atenção Básica. Vigilância em Saúde: dengue, esquistossomose, hanseníase, malária, tracoma e tuberculose. Brasília: Ministério, 2008a.

BRASIL. Ministério da Saúde. Secretaria de Vigilância em Saúde. Departamento de Vigilância Epidemiológica. Manual nacional de vigilância laboratorial da tuberculose e outras micobacterioses. Brasília, 2008b.

BRASIL. Ministério da Saúde. Manual de Recomendações para o Controle da Tuberculose no Brasil. 1. ed. Brasília: Ministério da Saúde; 2011.

BRASIL. Ministério da Saúde. Perspectivas brasileiras para o fim da tuberculose como problema de saúde pública. Boletim epidemiológico, v. 47, n. 13, p. 1-15, 2016.

BRASIL. Ministério da Saúde. Manual de Recomendações para o Controle da Tuberculose no Brasil. 2. ed. Brasília: Ministério da Saúde; 2019a.

BRASIL. Ministério da Saúde. Secretaria de Vigilância em Saúde. Boletim Epidemiológico, v. 50, n. 09. Mar. de 2019b.

BRASIL. Exame de baciloscopia para tuberculose. Portal da Internet: Governo do Brasil. 2019c. Disponível em: <Exame de Baciloscopia para Tuberculose 0 Português (Brasil) (www.gov. br)> Acesso em: 10 de mai. 2021

BRASIL. Ministério da Saúde. Secretaria de Vigilância em Saúde. Boletim Epidemiológico Tuberculose 2020. Brasília: Ministério da Saúde; 2020a. Número Especial. Mar. 2020.

BRASIL. Portal da Saúde. Brasília: Ministério da Saúde. Tuberculose no Brasil e no mundo. 2020b. Disponível em: <http://portal.saude.gov.br/portal/saude/profissional/visualizar_texto.cfm?idtxt=31109> Acesso em: 07 de nov. de 2020
BRASIL. Ministerio da Saude. Secretaria de Vigilancia em Saude. Plano de

Contingencia Nacional para Infeccao Humana pelo Novo Coronavirus COVID-19. Brasilia: Ministerio da Saude, 2020c Disponivel em: <https://

portalarquivos2.saude.gov.br/images/pdf/2020/fevereiro/13/planocontingencia-

coronavirus-COVID19.pdf>. Acesso em: 04 mai. 2021.

BRASIL. Ministério da Saúde. Secretaria de Vigilância em Saúde. Boletim Epidemiológico Tuberculose 2021. Brasília: Ministério da Saúde; 2021. Número Especial. Mar. 2021.

BRAUNWALD, E.; KASPER, D.L.; FAUCI, A.S.; JAMESON, J.L.; Longo, D.L.; HAUSER S. Harrison's Principles of Internal Medicine. 18 ed. NY: McGraw-Hill; 2013.

CARDONA, P.-J. Patogénesis de la tuberculosis y otras micobacteriosis, 2018;36(1), 38-46.

CDC. Chapter 2 Transmission and Pathogenesis of Tuberculosis. (2012). Chapter 2 Transmission and Pathogenesis of Tuberculosis, 19-44. Disponível em: https://www.cdc.gov/tb/ education/corecurr/pdf/chapter2.pd>Acesso em: 10 mai 2021

CONDE, M.B.; MELO, F.A.; MARQUES, A.M.; CARDOSO, N.C; PINEHIRO, V.G.; DALCIN, Pde T.; et al. III Brazilian Thoracic Association Guidelines on tuberculosis. J Bras Pneumol 2009;35(10):1018-48.

CORTEZ, A. O.; MELO, A. C.; NEVES, L. O.; RESENDE, K. A; CAMARGOS, P. Tuberculose no Brasil: um país, múltiplas realidades. J Bras Pneumol. 2021;47(2):e20200119. DOI: https://dx.doi.org/10.36416/1806-3756/e20200119

COURA, J. R. Dinâmica das doenças infecciosas e parasitárias. In: Dinâmica das doenças infecciosas e parasitárias. 2 ed. ed. Rio de Janeiro: Guanabara Koogan, 2013.

DANIEL, T. M. The original and precolonial epidemiology of tuberculosis in the Americas: can we figure them out?Int ] Tuberc Lung Dis. 2000;4(5):395-400. 10815731.

FOUNDATION FOR INNOVATIVE NEW DIAGNOSTICS (FIND) 2020. Frequently Asked Questions on Xpert MTB/RIF assay. Disponível em: <http://www.finddiagnostics.org/export/ sites/default/media/press/pdf/Xpert_FAQs.pdf> Acesso em: 13 nov 2020 .

HIJJAR, M.A.; PROCÓPIO, M.J. Tuberculose: epidemiologia e controle no Brasil. Ano 5, julho/ dezembro de 2006.

JACOMELLI, M.; ARAÚJO, P.; RODRIGUES, A.J.; DEMARZO, S.E.; SEICENTO, M.; FIGUEIREDO, V.R. Broncoscopia no diagnóstico de tuberculose pulmonar em pacientes com baciloscopia de escarro negativa. J Bras Pneumol. 2012;38(2):167 173. 
LABORCLIN. Coloração Ziehl-Neelsen. Rev. 12- 11/2019. Instrução de Uso do Fabricante. Disponível em: <https://www. laborclin.com.br/wp-content/uploads/2019/05/620522-COLORACAO-ZIEHL-NEELSEN-CONJ-3x100mL.pdf> Acesso em: 16 de nov. de 2020.

MACIEL, E. L.; JUNIOR, E. G.; DALCOLMO, M. M. Tuberculose e coronavírus: o que sabemos? Epidemiol Serv Saúde 29(2):e2020128, 2020. DOI: https://doi.org/10.5123/S167949742020000200010

MACIEL, M.S.; MENDES, P.D.; GOMES, A.P.; BATISTA, R.S. A história da tuberculose no Brasil: os muitos tons de cinza da miséria. Revista Brasileira Clin Med. São Paulo, 2012 mai-jun; 10(3): 226-30

MARLOWE, E.M.; NOVAK-WEEKLEY, S.M.; CUMPIO, J.; SHARP, S.E.; MOMENY, M.A.; BABST, A. Evaluation of the cepheid Xpert MTB/RIF assay for direct detection of Mycobacterium tuberculosis complex in respiratory specimens.J Clin Microbiol, 49: 1621-1623, 2011.

MARQUES, C. C. et al.Casos de tuberculosis coinfectados por VIH en el estado del noreste brasileño. Enfermería Actual de Costa Rica. 2019;(36):62-76. DOI: <http://dx.doi. org/10.15517/revenf.v0i36.33583>

MELLO, F. C. Abordagem diagnóstica da tuberculose pulmonar. Pulmão RJ 2012(1):27-31. Disponível em: <revistacompleta_2012_1.pdf (sopterj.com.br)> Acesso em: 07 nov. 2020

NASCIMENTO, D.R. As pestes do século XX: tuberculose e AIDS no Brasil, uma história comparada. Rio de Janeiro: Editora Fiocruz; 2005.

OLIVEIRA, M.F., ARCÊNIO, R.A., RUFFINO-NETO, A., SCATENA, L.M., PALHA, P.F., VILLA, T.C.S. A porta de entrada para o diagnóstico da tuberculose no sistema de saúde de Ribeirão Preto/SP. Esc Enferm USP. 2010

OLIVEIRA, M. S.; et al. Perfil epidemiológico dos casos de tuberculose no estado do Maranhão nos anos de 2012 a 2016. Rev Pre Infec e Saúde[Internet]. 2018;4:6896. DOI: https://doi.org/10.26694/repis.v4i0.6896

PATEL, V.B.; THERON, G.; LENDERS, L.; MATINYENA, B.; CONNOLLY, C. SINGH, R. Diagnostic accuracy of quantitative PCR (Xpert MTB/RIF) for tuberculous meningitis in a high burden setting: a prospective study. PLoS Med, 10: 2013

PINTO, L. M.; PAI, M.; DHEDA, K.; SCHWARTMAN, K.; MENZIES, D.; STEINGART, K. R. Scoring systems using chest radiographic features for the diagnosis of pulmonay tuberculosis in adults: A systematic review. Europen Respiratory Journal, Clinical Microbiology and Infection. Vol. 8, № 6 - Nov/ Dez, 2013, p 235-243.

SANTOS, D. A.; MARQUES, A. L.; GOULART, L. S.; MATTOS, M.; OLINDA, R. A. Fatores associados ao abandono do tratamen- to da tuberculose pulmonar. Cogitare enferm. 2021, v26:e72794. DOI:https://dx.doi.org/10.5380/ce.v26i0.72794

STATENS SERUM INSTITUT. Tuberculina PPD RT 23 para Teste de Mantoux. Master diagnóstica. Instrução de Uso do Fabricante. Disponível em: <http://masterdiagnostica.com. br/produto/ppd-rt-23-ssi-tuberculina> Acesso em: 16 de nov. de 2020.

SILVA, D. R.; MELLO, F. C.; D’AMBROSIO, L.; CENTIS, R.; DALCOLMO, M. P.; MIGLIORI, G. B. Tuberculose e COVID-19, o novo dueto maldito: quais as diferenças entre Brasil e Europa? J Bras Pneumol. 2021a ;47(2):e20210044.DOI: https://dx.doi. org/10.36416/1806-3756/e20210044

SILVA, D. R., et al. Consenso sobre o diagnóstico da tuberculose da Sociedade Brasileira de Pneumologia e Tisiologia. 2021b ;47(2):e20210054. DOI: https://dx.doi.org/10.36416/1806$3756 / \mathrm{e} 20210054$

SILVA, P. D. Tuberculose no Tocantins: perfil epidemiológico dos casos de retratamento e fatores associados à recidiva e ao abandono. 2017; 64 f. Dissertação (Mestrado em Saúde Coletiva) - Instituto de Saúde Coletiva, Universidade Federal da Bahia, Salvador

SOTGIU, G.; NAHID P.; LODDENKEMPER, R.; ABUBAKAR, I.; MIRAVITLLES, M.; MIGLIORI, G. B. The ERS-endorsed official ATS/CDC/IDSA clinical practice guidelines on treatment of drug-susceptible tuberculosis. Eur RespirJ. 2016;48(4):963-

TAVARES, C.M.; CUNHA, A.M.S.; GOMES, N.M.C.; LIMA, A.B.A.; SANTOS, I.M.R.; ACÁCIO, M.S. et al. Tendência e caracterização epidemiológica da tuberculose em Alagoas, 2007. 2016. Cad Saúde Colet, 2020;28(1):107-115.

TEIXEIRA, A.Q.; SAMICO, I.C.; MARTINS, A.B.; GALINDO, J.M.; MONTENEGRO, R.A.; SCHINDLER, H.C. Tuberculose: conhecimento e adesão às medidas profiláticas em indivíduos contatos da cidade do Recife, Pernambuco, Brasil. Cad Saúde Colet., 2020;28(1):116-129

VERONESI, R.; FOCACCIA, R. Tratado de Infectologia. 4. ed. Rio de Janeiro: Atheneu; 2010.

VERONESI, R.; FOCACCIA, R. Tratado de Infectologia. 5. ed. rev. e atual. São Paulo: Editora Atheneu; 2015.

WORLD HEALTH ORGANIZATION (WHO). Draft global strategy and targets for tuberculosis prevention, care and control after 2015. [A67/11] Secretariat World Heath Assembly, 2014. Disponível em: <http://apps.who.int/gb/ebwha/pdf_files/WHA67/A67_11en.pdf>. Acesso em: 24 de nov. de 2020.

WORLD HEALTH ORGANIZATION (WHO). Fact sheets: Tuberculosis, 2020a. Disponível em: <https://www.who.int/news-room/ fact-sheets/detail/tuberculosis> Acesso em: 31 de ago. de 2020. 
WORLD HEALTH ORGANIZATION (WHO). Global tuberculosis control: epidemiology, strategy, financing. Geneva, 2009.

WORLD HEALTH ORGANIZATION. Global Tuberculosis Report 2016. Geneva: WHO; 2016.

WORLD HEALTH ORGANIZATION. Global Tuberculosis Report 2017. Geneva: WHO; 2017.
WHO, WORLD HEALTH ORGANIZATION (WHO). Implementing tuberculosis diagnostics: policy framework. 2015.

WORLD HEALTH ORGANIZATION. Global Tuberculosis Report 2018. Geneva: WHO; 2018.

WORLD HEALTH ORGANIZATION. Global Tuberculosis Report 2019. Geneva: WHO; 2019.

WORLD HEALTH ORGANIZATION. Global Tuberculosis Report 2020. Geneva: WHO; 2020b. 